\title{
A importância de uma base de dados na gestão de serviços de saúde
}

\author{
The importance of a database in the management of healthcare services
}

\author{
Cláudia Toledo de Andrade ${ }^{1}$, Ana Maria Prado Castello Branco Magedanz ${ }^{2}$, Daísa Mesquita Escobosa², \\ Wellington Martins Tomaz ${ }^{2}$, Carolina Sampieri Santinho ${ }^{2}$ Tania Oliveira Lopes ${ }^{2}$, Valéria Lombardo ${ }^{3}$
}

\begin{abstract}
RESUMO
Objetivo: Apresentar o perfil epidemiológico de pacientes com câncer pertencentes à base de dados do Registro Hospitalar de Câncer e relatar a importância dessa base para a gestão de serviço de saúde de um Centro de Oncologia e Hematologia. Métodos: Estudo retrospectivo, realizado com levantamento de 1.617 prontuários, entre os anos de 2004 a 2009. Os cânceres analisados foram de cólon e reto, mama, próstata e pulmão. As variáveis utilizadas no estudo foram idade, gênero, estadiamento da doença ao diagnóstico e tipo de tratamento. Para a coleta de dados, utilizou-se o software SisRHC e, na análise, utilizou-se o software Excel ${ }^{\circledR}$. Para a segurança e confiabilidade dos dados, foram utilizadas senhas de acessos aos arquivos, backups semanais das planilhas, além do CPF do paciente, a fim de evitar duplicidade. Resultados: Do total de 1.617 prontuários analisados, $36,42 \%$ pertenciam ao grupo topográfico de mama e aproximadamente $40 \%$ dos pacientes com câncer de cólon e reto tinham 70 anos ou mais. 0 estadiamento II foi prevalente, representando 44\% da amostra, e o tratamento mais empregado foi a cirurgia ( $57 \%$ dos casos). Conclusão: 0 presente estudo sugeriu que a base de dados do Registro Hospitalar de Câncer tem papel relevante por ser capaz de gerar um conjunto de importantes informações relacionadas ao câncer e que, por meio dessas informações, é possível realizar estudos epidemiológicos que permitam gerar ações de melhoria gerencial e assistencial a essa população.
\end{abstract}

Descritores: Neoplasias; Base de dados; Gestão em saúde

\section{ABSTRACT}

Objective: To present the epidemiological profile of cancer patients belonging to a database of a Hospital Cancer Registry and to report on the importance of this database in managing healthcare services at an Oncology and Hematology Center. Methods: A retrospective survey conducted with 1617 records of patients diagnosed with cancer at the institution between 2004 and 2009. The types of cancer analyzed were of the colon and rectum, breast, prostate and lung. The variables used in the study were age, gender, stage of disease upon diagnosis and treatment. Researchers used an application called SisHCR for data collection, and Excel ${ }^{\circledR}$ for data analysis. To ensure data safety and reliability, passwords were required for accessing files, spreadsheets were backed up on a weekly basis, and patients' CPF numbers were used to avoid data duplication. Results: Of the 1617 records analyzed, $36.42 \%$ belonged to the topographic group of the breast, and approximately $40 \%$ of patients with colorectal cancer were 70 years of age or older. Stage II was prevalent, representing $44 \%$ of the sample, and the treatment most used was surgery $(57 \%$ of cases). Conclusion: The study suggested that the Hospital Cancer Registry database is an instrument capable of generating important information about cancer, and that through this information, epidemiological studies can be carried out and the processes of management and care can be improved.

Keywords: Neoplasms; Database; Health management

\section{INTRODUÇÃO}

De acordo com o relatório da Agência Internacional para Pesquisa sobre Câncer (do inglês International Agency for Research on Cancer - IARC)/Organização Mundial da Saúde (OMS), World Cancer Report 2008, o impacto global das neoplasias, em 30 anos, mais que dobrou. Estimou-se que, nos últimos anos, ocorreriam no mundo cerca de 12 milhões de novos casos de câncer e 7 milhões de óbitos ${ }^{(1)}$.

\footnotetext{
Trabalho realizado no Centro de Oncologia e Hematologia, Hospital Israelita Albert Einstein - HIAE, São Paulo (SP), Brasil.

${ }^{1}$ Serviço de Prática Assistencial, Centro de Oncologia e Hematologia, Sociedade Beneficente Israelita Brasileira Hospital Albert Einstein - SBIBHAE, São Paulo (SP), Brasil.

${ }^{2}$ Registro Hospitalar de Câncer, Hospital Israelita Albert Einstein - HIAE, São Paulo (SP), Brasil.

${ }^{3}$ Registro Hospitalar de Câncer do Estado de São Paulo, Fundação Oncocentro de São Paulo - FOSP, São Paulo (SP), Brasil.

Autor correspondente: Cláudia Toledo de Andrade - Avenida Albert Einstein, 627/701 - Morumbi - CEP: 05651-901 - São Paulo (SP), Brasil - Tel.: (11) 2151-2004 - Email: ct_andrade@hotmail.com

Data de submissão: 11/5/2011 - Data de aceite: 4/7/2011
}

Conflitos de interesse: não há. 
No Brasil, as estimativas para os anos de 2010 e 2011 aproximam-se de 489.270 casos novos de câncer, sendo os mais frequentes o de próstata e o de pulmão para o gênero masculino, e o de mama e o de colo do útero para o feminino ${ }^{(1)}$.

Dessa forma, tornam-se fundamentais investimento e desenvolvimento de ações de melhoria do controle do câncer, por meio de detecção precoce, vigilância dos fatores de risco e pesquisa. A partir dos registros e de dados fidedignos, é possível realizar análises que são base para uma adequada tomada de decisão gerencial e assistencial ${ }^{(2,3)}$.

Para implementar e executar ações específicas, é fundamental a existência dos Registros Hospitalares de Câncer (RHC) e os Registros de Câncer de Base Populacional (RCBP), os quais são responsáveis por reportar informações atualizadas, de qualidade sobre câncer e que representem a população. Esses registros são considerados pelo Instituto Nacional de Câncer (INCA) como "ferramentas poderosas para a vigilância epidemiológica do câncer no país"(4).

Para acompanhar e analisar informações de câncer, há, no Estado de São Paulo, a Fundação Oncocentro de São Paulo (FOSP), instituição pública vinculada à Secretaria Estadual da Saúde, que assessora a formulação e a condução de políticas de saúde na área de Oncologia no Estado e serve como instrumento para que os hospitais oncológicos possam formular seus protocolos e melhorar suas práticas assistenciais ${ }^{(5)}$.

Os principais objetivos da FOSP são registrar novos casos de câncer e o seguimento dos pacientes, para acompanhar a evolução da doença, e empreender estudos epidemiológicos nessa área ${ }^{(6)}$.

Para isso, a FOSP utiliza um software denominado Sistema de Registro Hospitalar de Câncer (SisRHC), o qual é disponibilizado aos 75 Centros de Alta Complexidade em Oncologia e 5 hospitais voluntários, que repassam suas informações a essa instituição ${ }^{(5,6)}$.

Os hospitais que colaboram com a FOSP possuem um setor denominado RHC, que é responsável por transmitir informações sobre câncer à FOSP e à própria instituição à qual pertencem ${ }^{(7)}$.

Por meio dos dados do RHC, é possível conhecer a assistência oferecida ao paciente oncológico e, dessa forma, melhorar a atenção assistencial dispensada a essa população. Além disso, esses dados podem ser utilizados como fonte de informações para planejamento administrativo, pesquisas clínicas e efetuar um controle eficiente do seguimento dos pacientes atendidos, possibilitando a análise de sobrevida dos mesmos.

Outra contribuição é que o RHC permite a troca de informações com outros registros hospitalares, bem como a organização de estudos colaborativos que pos- sam caracterizar um tipo de câncer numa população de forma mais ampla.

Diante do exposto, o RHC torna-se um importante instrumento para estudos epidemiológicos, além de ser um recurso que permite analisar a eficiência e a eficácia dos procedimentos diagnósticos e terapêuticos do câncer, conjuntamente com a avaliação de desfechos, tais como sobrevida e recidivas.

\section{OBJETIVO}

Os objetivos deste estudo foram: apresentar o perfil epidemiológico de pacientes com câncer, pertencentes à base de dados do RHC de um Centro de Oncologia e Hematologia, e relatar a importância de tal base de dados para gestão de serviço de saúde nesse tipo de centro.

\section{MÉTODOS}

\section{Tipo de estudo}

Estudo retrospectivo, de caráter descritivo exploratório, nível I, com análise quantitativa dos dados. A pesquisa quantitativa, descritiva e exploratória é aquela na qual se coletam descrições detalhadas de variáveis existentes e usa-se o dado para justificar e avaliar condições práticas de atenção ${ }^{(8,9)}$.

\section{Campo de estudo}

O estudo foi desenvolvido no Centro de Oncologia e Hematologia de um hospital geral, de grande porte, localizado no Município de São Paulo.

\section{População e amostra}

A população foi formada por pacientes com câncer de cólon e reto, mama, próstata e pulmão. $\mathrm{O}$ período analisado esteve compreendido entre 2004 a 2009.

Os pacientes chegaram à instituição com os seguintes status: (1) sem diagnóstico e sem tratamento; (2) com diagnóstico e sem tratamento; (3) com diagnóstico e com tratamento.

As variáveis analisadas foram: gênero, idade, estadiamento da doença ao diagnóstico e tipo de tratamento.

\section{Instrumento de coleta de dados}

Para a coleta de dados, os pesquisadores utilizaram o software SisRHC e, para análise de dados, o Excel ${ }^{\circledR}$.

Para segurança e confiabilidade do banco de dados, foram criadas senhas de acesso aos arquivos, garantindo-se a utilização dos dados somente pelos pesquisadores autorizados. Realizaram-se backups semanais das 
planilhas, e os arquivos ficaram armazenados em rede de acesso restrito.

Para determinar o grau de disseminação dos tumores, os pesquisadores utilizaram o sistema TNM. Esse sistema, desenvolvido pela American Joint Committee on Cancer (AJCC) e International Union Against Cancer (UICC), determina o estadiamento dos tumores sólidos medindo o tamanho do tumor $(\mathrm{T})$, verificando se a doença acometeu algum linfonodo $(\mathrm{N})$ e se há metástase à distância $(\mathrm{M})^{(10)}$.

\section{Operacionalização da coleta de dados}

A coleta ocorreu entre $1^{\mathrm{o}}$ de fevereiro de 2012 e 24 de março de 2011, após aprovação do projeto de pesquisa pelo Comitê de Ética em Pesquisa (CEP) do Hospital Israelita Albert Einstein (HIAE), sob o número 1.497. Por se tratar de um trabalho não intervencional, retrospectivo, em prontuário e com obrigatoriedade de sigilo, solicitamos ao CEP a não utilização do Termo de Consentimento Livre e Esclarecido (TCLE).

Os autores comprometeram-se em utilizar as informações obtidas exclusivamente nesta pesquisa, bem como a manter sob sigilo a identificação dos sujeitos, segundo a resolução 96/1996.

\section{Análise e apresentação dos resultados}

Os dados foram apresentados em números absolutos e percentuais, sob a forma de tabelas e gráficos.

Na construção do banco de dados RHC, utilizamos o número do $\mathrm{CPF}$ do paciente e o número do prontuário, garantindo, assim, a não duplicidade dos dados.

\section{RESULTADOS}

\section{Incidência}

Analisamos uma amostra de 1.617 prontuários de pacientes. Desse total, $191(11,81 \%)$ pertenciam ao grupo de pulmão, 327 (20,22\%) cólon e reto, $510(31,53 \%)$ eram de próstata e $589(36,42 \%)$ eram de mama, conforme apresentado na tabela 1.

Tabela 1. Distribuição do número de pacientes, por topografia, entre os anos de 2004 e 2009

\begin{tabular}{lccc}
\hline Topografia & $\begin{array}{c}\text { Pacientes } \\
\mathbf{n}(\%)\end{array}$ & $\begin{array}{c}\text { Média de } \\
\text { pacientes/ano }\end{array}$ & Desvio-padrão \\
\hline Mama & $589(36)$ & 98 & 42 \\
Próstata & $510(32)$ & 85 & 34 \\
Cólon e reto & $327(20)$ & 55 & 24 \\
Pulmão & $191(12)$ & 32 & 12 \\
\hline Total & $1.617(100)$ & - & - \\
\hline
\end{tabular}

A maior incidência relacionou-se ao câncer de mama, o qual representou quase $40 \%$ do total da amostra. A segunda doença mais observada foi o câncer de próstata com $32 \%$.

\section{Gênero}

No gênero feminino, destaca-se o câncer de mama como a doença mais frequentemente e, para o masculino, próstata foi o câncer mais incidente, conforme demonstrado na tabela 2.

Tabela 2. Distribuição dos gêneros masculino e feminino dentro das quatro topografias

\begin{tabular}{lccc}
\hline \multirow{2}{*}{ Topografia } & \multicolumn{2}{c}{ Gênero } & \multirow{2}{*}{ Total (\%) } \\
\cline { 2 - 3 } & Feminino n (\%) & Masculino n(\%) & \\
\hline Mama & $582(98,81)$ & $7(1,19)$ & 100 \\
Próstata & - & $510(100)$ & 100 \\
Cólon e reto & $152(46,48)$ & $175(53,52)$ & 100 \\
Pulmão & $75(39,27)$ & $116(60,73)$ & 100 \\
\hline
\end{tabular}

Tanto para o câncer de cólon e reto quanto para o de pulmão, a maior incidência foi evidenciada no gênero masculino, com 53,52 e $60,73 \%$, respectivamente.

\section{Faixa etária}

A tabela 3 apresenta o número de pacientes distribuídos de acordo com faixas etárias. Observa-se alta incidência de câncer de cólon e reto na faixa etária de 70 anos ou mais, com 130 pacientes, o que representa quase $40 \%$ dos 327 pacientes com esta doença.

Tabela 3. Número de pacientes com câncer de mama, próstata, cólon e reto, e pulmão, de acordo com faixas etárias

\begin{tabular}{lccccc}
\hline $\begin{array}{l}\text { Faixa } \\
\text { etária }\end{array}$ & Mama & Próstata & $\begin{array}{c}\text { Cólon e } \\
\text { reto }\end{array}$ & Pulmão & Total (\%) \\
\hline $0-9$ & 0 & 0 & 0 & 0 & $0(0)$ \\
$10-19$ & 0 & 0 & 1 & 0 & $1(0,1)$ \\
$20-29$ & 1 & 0 & 2 & 1 & $4(0,2)$ \\
$30-39$ & 54 & 0 & 8 & 4 & $66(4,1)$ \\
$40-49$ & 168 & 20 & 37 & 12 & $237(14,7)$ \\
$50-59$ & 165 & 160 & 65 & 34 & $424(26,2)$ \\
$60-69$ & 103 & 220 & 84 & 65 & $472(29,2)$ \\
$70+$ & 98 & 110 & 130 & 75 & $413(25,5)$ \\
\hline Total & 589 & 510 & 327 & 191 & $1.617(100)$ \\
\hline
\end{tabular}




\section{Estadiamento}

Conforme figura 1 , o estádio II foi o mais incidente, totalizando $44 \%$ da amostra. O estádio I teve $24,7 \%$, o III foi de $15 \%$, o IV teve $10 \%$, in situ com $5,8 \%$ e o Y representou $0,5 \%$ dos casos analisados.

A curva de sobrevida apresentada na figura 2 demonstra a importância entre o reconhecimento precoce da doença e seu desfecho. Ela mostra, afinal, que, quanto mais tempo se demora para chegar ao diagnóstico, menos tempo de sobrevida pode ter o paciente ${ }^{(11)}$.

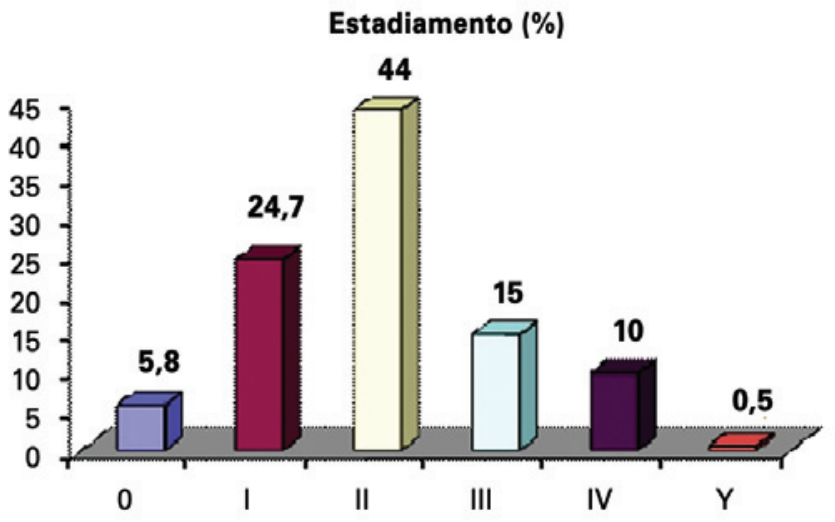

Figura 1. Apresentação dos estadiamentos dos quatro tipos de tumores analisados na amostra, segundo estádio clínico. Estádio 0: in situ; estádio Y: tumores não classificados segundo TNM

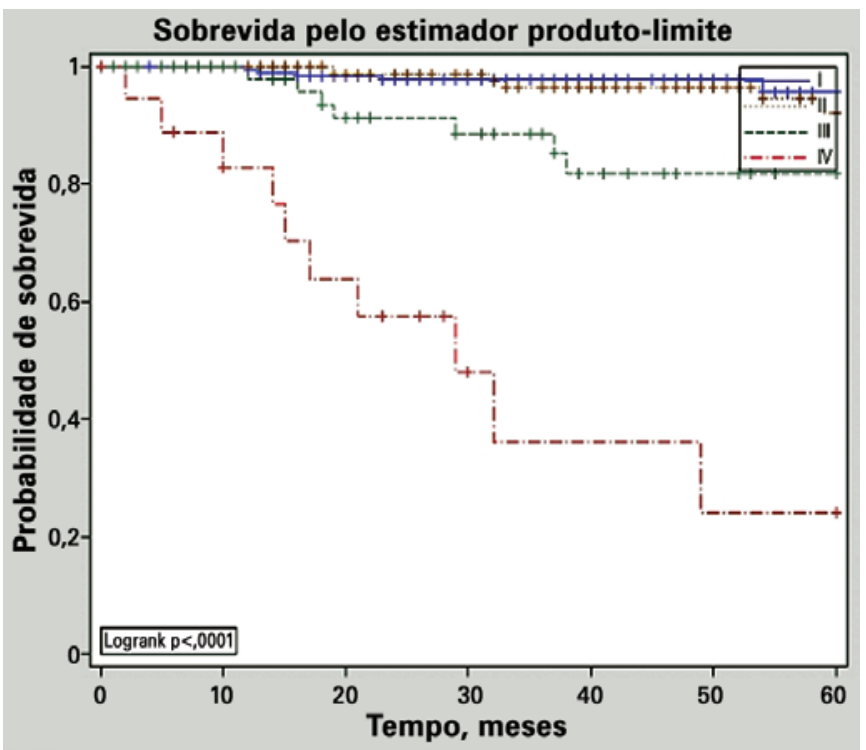

Figura 2. Curva de sobrevida de Kaplan - Mama 2009. Sobrevida (\%) de casos de tumores de mama, segundo estádio clínico e tempo em meses

\section{Tratamentos}

De acordo com figura 3, dentre os tratamentos mais empregados, destacam-se: cirurgia como primeiro tratamento de escolha em 57\% dos casos, seguidos de $13 \%$

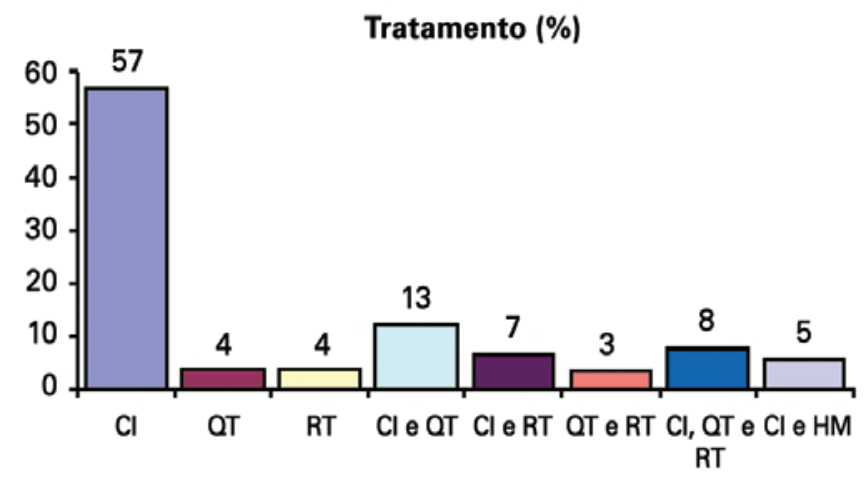

Cl: cirurgia; QT: quimioterapia; RT: radioterapia; HM: hormonioterapia.

Figura 3. Tipos de tratamento empregados no combate aos cânceres de mama, próstata, cólon e reto, e pulmão

que receberam a combinação de cirurgia e quimioterapia. O terceiro tratamento mais empregado foi a combinação entre cirurgia, quimioterapia e radioterapia.

\section{DISCUSSÃO}

Na saúde, a utilização de um sistema de apoio à tomada de decisões gerencial e assistencial pode ser de grande auxílio, pois permite analisar dados por meio do cruzamento de informações, relacionando problemas de saúde com seus fatores determinantes e identificando os riscos para o acometimento de doenças ${ }^{(12)}$.

Tais indicadores deverão servir de elementos para a execução de ações de prevenção na melhoria da qualidade de vida dos pacientes ${ }^{(11)}$.

Conforme constatado em outros estudos da área de saúde, a utilização de sistemas de informação torna-se fundamental, no sentido de acompanhar e oferecer respostas às novas e complexas demandas consequentes das transformações organizacionais.

Um estudo realizado no Município de Ribeirão Preto (SP) teve como objetivo desenvolver um sistema de auxílio à tomada de decisão sobre os diagnósticos e intervenções de enfermagem em vítimas de trauma no atendimento avançado pré-hospitalar ${ }^{(13)}$. Os resultados desse estudo indicaram que as informações armazenadas corretamente, bem como o tempo de retorno, foram ideais, tanto para a consulta do diagnóstico como para a consulta de intervenções. Concluiu-se que a implementação do sistema otimizou o tempo despendido para a elaboração do diagnóstico de enfermagem de clientes vítimas de trauma ${ }^{(13)}$.

Por meio de análise das informações extraídas da base de dados do RHC, identificou-se, por exemplo, a alta incidência de câncer de cólon e reto na faixa etária de 70 anos ou mais, o que pode ser explicado pelo fato desse câncer ter um desenvolvimento lento e silencioso. 
Dessa forma, o diagnóstico é habitualmente feito em idades mais elevadas e, consequentemente, o estádio é mais avançado, piorando significativamente o prognóstico do paciente ${ }^{(14)}$.

Também caracterizou-se o estádio II como o mais incidente dentre os pacientes analisados. Os cânceres com estádio I são os menos avançados e, geralmente, têm um melhor prognóstico; os de estádio III e IV são mais avançados, porém, em muitos casos, também podem ser tratados com sucesso ${ }^{(10)}$.

Em relação à eficácia, o tratamento inicial de câncer é maior nos pacientes com estádios mais avançados da doença. O custo de tratamento de tumores metastáticos é aproximadamente o dobro se comparado ao que se gasta com aqueles pacientes diagnosticados com doença local ${ }^{(15)}$.

Para a gestão gerencial e assistencial de um serviço ou hospital especializado em oncologia é de extrema importância conhecer o perfil epidemiológico de sua população. Nesse contexto, possuir uma base de dados fidedigna torna-se de grande auxílio para a tomada de decisão, por exemplo, sobre os tipos de tratamentos que serão oferecidos e a instauração de campanhas de detecção precoce do câncer, além de servir como base para planejamento da própria estrutura física.

A fim de transmitir dados mais precisos, o RHC da do HIAE realiza um trabalho dinâmico de coleta de dados, os quais são advindos de seis diferentes fontes, conforme figura 4 .

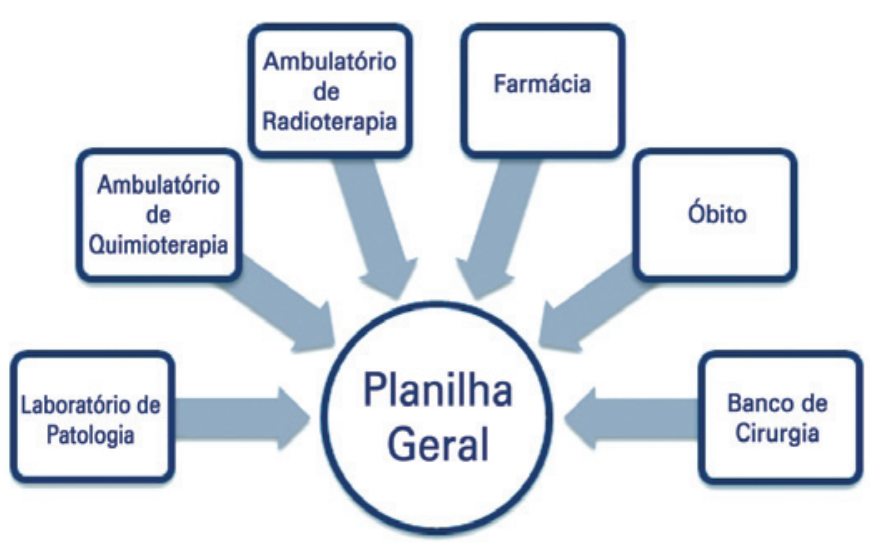

Figura 4. Demonstração do fluxo de captação de pacientes oncológicos para o Registros Hospitalares de Câncer

Após a criação da planilha geral, os dados foram analisados e tabulados pelo software SisRHC, da FOSP, e, a partir daí, foi possível realizar estudos sobre os diferentes tipos de cânceres.

As informações geradas pelo RHC são transmitidas periodicamente ao comitê gestor do Centro de
Oncologia e Hematologia da instituição, a fim de informar e apoiar a melhoria contínua dos processos gerencial e assistencial desse segmento da saúde.

As melhorias implementadas por meio das informações extraídas do RHC justificam a importância da existência de uma base de dados que auxilie na gestão de serviço de saúde de um Centro de Oncologia e Hematologia.

Por esses motivos e como complementação deste estudo, será desenvolvido um segundo estudo, buscando fortalecer a importância dessa ferramenta gerencial e descrever as melhorias dos processos implementadas no RHC do HIAE.

\section{CONCLUSÃO}

O presente estudo sugeriu que as informações da base de dados do RHC têm papel relevante para os diversos serviços e hospitais oncológicos, por ser capaz de gerar um conjunto de importantes informações relacionadas ao câncer.

Por meio dessas informações, é possível realizar estudos epidemiológicos e utilizá-los como recursos que permitam analisar, por exemplo, a qualidade da assistência prestada aos pacientes oncológicos, o que, consequentemente, pode gerar ações de melhoria da atenção oferecida a essa população.

Além disso, com a utilização dos resultados obtidos nessa base de dados, é possível obter informações capazes de auxiliar no planejamento administrativo e, assim, melhorar a gestão do serviço de saúde da instituição.

Assim, conclui-se que uma base de dados oncológica é de extrema importância para os processos de gestão gerencial e assistencial, desde que suas informações sejam coletadas, tabuladas, armazenadas e monitoradas com segurança.

\section{REFERÊNCIAS}

1. Brasil. Ministério da Saúde. Secretaria Nacional de Assistência à Saúde Instituto Nacional do Câncer. Estimativa 2010: incidência de câncer no Brasil [Internet]. Rio de Janeiro: INCA; 2010 [citado 2010 Sep 1]. Disponível em: http://www.inca.gov.br/estimativa/2010/

2. Stacey D, Bennett CL, Barry MJ, Col NF, Eden KB, Holmes-Rovner M, et al. Decision aids for people facing health treatment or screening decisions. Cochrane Database Syst Rev. 2011;(10):CD001431.

3. Brennan S, McKenzie J, Whitty P, Buchan H, Green S. Continuous quality improvement: effects on professional practice and healthcare outcomes (Protocol for a Cochrane Review). In: The Cochrane Library, Issue 05, 2012.

4. Instituto Nacional de Câncer José Alencar Gomes da Silva. Coordenação Gera de Ações Estratégicas. Coordenação de Prevenção e Vigilância. Estimativa 2012: incidência de câncer no Brasil/Instituto Nacional de Câncer José Alencar Gomes da Silva, Coordenação Geral de Ações Estratégicas,Coordenação de Prevenção e Vigilância. Rio de Janeiro: INCA, 2011. 
5. Fundação Oncocentro de São Paulo (FOSP). Fundação Oncocentro de São Paulo: história [Internet]. São Paulo: FOSP; 2010 [citado 2010 Jun 16]. Disponível em: http://www.fosp.saude.sp.gov.br/quem_somos.html\#

6. Brasil. Ministério da Saúde. Secretaria Nacional de Assistência à Saúde. Instituto Nacional do Câncer. Registros hospitalares de câncer: rotinas e procedimentos [Internet]. Rio de Janeiro: INCA; 2000 [citado $2011 \mathrm{Abr}$ 25]. Disponível em: http://www1.inca.gov.br/17148F20-F49A-4918-9D48089E6F1F1202/FinalDownload/Downloadld-11A1AFB8520F0FB8BC755D0C 70B3819F/17148F20-F49A-4918-9D48-089E6F1F1202/vigilancia/download/ manual_rotinas_procedimentos_rhc.pdf

7. Bergamasco VD, Marta GN, Kowalski LP, Carvalho, AL. Perfil epidemiológico do câncer de cabeça e pescoço no estado de São Paulo. Rev Bras Cir Cabeça Pescoço. 2008;37(1):15-9.

8. Lakatos EM, Marconi MA. Fundamentos de metodologia científica. $6^{a}$ ed. São Paulo: Atlas; 2007.

9. Polit DF, Beck CT, Hungler BP. Fundamentos de pesquisa em enfermagem: métodos, avaliação e utilização. $5^{\mathrm{a}}$ ed. Porto Alegre: Artmed; 2004.

10. Mackillop WJ, Groome PA, Gospodarowicz MK, O'Sullivan B. 0 papel do estadiamento do câncer na medicina baseada em evidências. In: Pollock RE,
Doroshow JH, Khayat D, Nakao A, O'Sullivan B. Manual de oncologia clínica da UICC. $8^{a}$ ed. São Paulo: Fundação Oncocentro de São Paulo; 2006.

11. Migowski A, Silva GA. Survival and prognostic factors of patients with clinically localized prostate cancer. Rev Saude Publica. 2010;44(2):344-52.

12. Morais E, Silva SS, Caritá EC. Business intelligence utilizando tecnologias web para análise de fatores de risco na ocorrência de doença arterial coronariana. J Health Inform. 2010:2(1):7-13.

13. Caritá EC, Nini RA, Melo AS. Sistema de auxílio aos diagnósticos de enfermagem para vítimas de trauma no atendimento avançado pré-hospitalar móvel utilizando as Taxonomias NANDA e NIC. J Health Inform. 2010:2(4):87-94.

14. Brasil. Ministério da Saúde. Secretaria de Assistência à Saúde. Instituto Nacional de Câncer. Falando sobre câncer do intestino [Internet] 2003. Rio de Janeiro: INCA; 2003 [citado 2011 Abr 25]. Disponível em: http://www. inca.gov.br/17148F20-F49A-4918-9D48-089E6F1F1202/FinalDownload/ Downloadld-F69796376718862D25518AAE20801D89/17148F20-F49A4918-9D48-089E6F1F1202/publicacoes/Falando_sobre_Cancer_de_Intestino.pdf

15. Yabroff KR, Lamont EB, Mariotto A, Warren JL, Topor M, Meekins A, et al. Cost of care for elderly cancer patients in the United States. J Natl Cancer Inst. 2008;100(9):630-41. 